\title{
Evaluation of Staging Laparoscopy in Colorectal Cancer Assessment
}

\author{
Ahmed Hassan*, Mohammed Elkerkary, Mohamed Gamal, Mahmoud \\ Gamal, Ahmed Gomaa, Islam Khaled \\ Department of General Surgery, Faculty of Medicine, Suez Canal University, Ismailia, Egypt
}

\begin{abstract}
Background: The goal of staging laparoscopy in primary gastrointestinal malignancies is to examine any local, regional, and/or metastatic disease in order to determine the stage of the presenting malignancy, and thus, the possibility of performing a curative resection. This study aimed to evaluate the role of staging laparoscopy in preoperative assessment of colorectal cancer patients. Subjects and Method: this is a cross-sectional study, conducted at Suez Canal University hospital. A total of 26 patients with colorectal cancer were enrolled and had a CT scanning with contrast and staging laparoscopy as a step in the preoperative assessment of their disease. Results: Staging laparoscopy of these patients revealed metastatic spread in 10 cases (38\%). Both pelvi-abdominal CT and staging laparoscopy had equally high specificity for metastatic diseases (99\%). However, regarding the sensitivity, staging laparoscopy was superior to pelvi-abdominal CT in detecting the presence of metastatic disease in general ( $83 \% \mathrm{vs} 66 \%$, $\mathrm{p}=0.026$ ). Conclusion: staging laparoscopy is a valuable tool in evaluating colorectal cancer patients preoperatively. Future studies should be conducted on a higher number of patients in order to detect the actual value of staging laparoscopy in detecting metastatic spread.
\end{abstract}

Key words: colorectal cancer, computed tomography, staging laparoscopy, metastasis

\section{Introduction}

Colorectal cancer is considered the most common malignancy of the gastrointestinal tract and it causes 600,000 deaths worldwide each year ${ }^{(1)}$. Following the diagnosis of colorectal cancer case, a thorough evaluation by clinical examination, laboratory tests and instrumental screening should be carried out in order to determine the presence or absence of metastatic disease ${ }^{(2)}$. Abdominal ultrasonog- raphy may be useful in detecting hepatic metastasis; however, a CT scanning is more helpful in these settings, especially in assessing the tumor-related complications such as fistula and obstruction. Yet, in the case of peritoneal spread, CT scanning has been reported to have relatively poor sensitivity $(3)$. On the other hand, laparoscopy is a minimally invasive procedure that is used to diagnose intra-abdominal diseases. It overcomes the limitations of radiological studies by enabling 
surgeons to directly inspect large surface areas of abdominal organs and obtain biopsies from suspicious lesions ${ }^{(4)}$. The goal of staging laparoscopy in primary gastrointestinal malignancies is to examine any local, regional, and/or metastatic disease in order to determine the stage of the presenting malignancy, and thus, the possibility of performing a curative resection(5). Several studies have compared the efficacy of staging laparoscopy in detecting metastatic spread in gastric cancer patients; however, and to our knowledge, this work is the first to evaluate the efficacy of the two modalities in colorectal cancer patients. Therefore, we evaluated the value of using staging laparoscopy in preoperative assessment of colorectal cancer patients.

\section{Patients and Methods}

\section{Study design and setting}

This prospective cross-sectional study was conducted at the surgical oncology unit in the department of general surgery of Suez Canal University hospital, Ismailia, Egypt. The study was approved by the medical ethics committee of Faculty of Medicine, Suez Canal University.

\section{Patients}

Twenty-six patients diagnosed with colorectal cancer were selected by a simple random technique. An informed consent was obtained from every patient. Exclusion criteria are listed in Box 1.

Box 1. Exclusion criteria in the present study

- Patients with complicated colorectal cancer presenting with intestinal obstruction.

- Patients with advanced coronary artery disease.

- Patients with mental and cognitive disorders.

- Patients with known extra-hepatic metastatic disease from cancer colon.

- Dense intra-abdominal from previous operation mainly surrounding the liver.

\section{Study Procedure}

Prior to the procedure, all patients were subjected to full medical history taking, which included their personal and demographic data, history of any medical illnesses, previous operations, and onset and duration of symptoms. General and abdominal examinations were then performed. Blood samples were withdrawn for complete blood count, coagulation profile (PT, PTT, INR), kidney function tests, liver enzymes and function tests (ALT, AST, GGT, alkaline phosphatase, total and direct bilirubin), viral markers (HBSAG and HCV AB), and tumor markers (CA19.9, CEA, AFP). Moreover, all pa tients had pelvi-abdominal ultrasound and $\mathrm{CT}$ scan. $\mathrm{CT}$ was used to determine the site and size of the tumor, nodal involvement, local infiltration and metastasis. Patients were finally requested to undergo colonoscopy to evaluate any suspicious masses and obtain a biopsy for histopathological evaluation. After these tests, all patients underwent diagnostic laparoscopy as a part of the staging process of their disease. Operable cases had a formal exploration, then resection of the pathological segment followed by anastomosis versus colostomy. Meanwhile, non-operable cases were referred to the oncology department. Following the procedure, patients 
were observed for any signs of complications, including drainage, bleeding, welling around incision sites, or mild fever, and patient were expected to resume their normal daily activities as early as possible.

\section{Statistical Analysis}

Data were analysed using Statistical Package for Social Sciences software (SPSS.21). Numerical data were expressed as mean \pm SD and categorical data as frequencies and percentages. Ttest was used to compare between quantitative data, while Chi-square was used to compare between the qualitative data. Results were considered statistically significant at a $\mathrm{p}$-value $<0.05$ and highly significant at $\mathrm{p}$-value $<0.01$.

\section{Results}

Patients were predominantly 50 years of age or older (81\%), females (54\%) and/or smokers (57\%) (Table 1). Colonoscopy was done as an essential step to diagnose colon cancer and multiple biopsies were taken from the suspicious lesions. Most of these biopsies ( $85 \%$ ) were positive for malignancy, whereas the rest (15\%) were non-specific biopsies requiring re-assessment to confirm the presence of malignancy (Figure 1). According to staging laparoscopy, liver was the most frequent site (80\%) for metastatic disease in colorectal cancer (Figure 2).

Table 1. Demographic characteristics of the studied patients $(\mathrm{N}=20)$

\begin{tabular}{|c|c|}
\hline Variables & $\mathrm{N}(\%)$ \\
\hline Age (years) Mean \pm SD & $56.7 \pm 9.0$ \\
$\geq 50$ Year & $21(81)$ \\
$<50$ Year & $5(19)$ \\
\hline Gender & \\
Male & $14(54)$ \\
Female & $12(46)$ \\
\hline Smoking & \\
Present & $15(57)$ \\
Absent & $11(43)$ \\
\hline
\end{tabular}

Table 2. Comparison between diagnostic laparoscopy and pelvi-abdominal $\mathrm{CT}$ in detecting metastasis $(\mathrm{N}=26)$

\begin{tabular}{|l|c|c|c|c|}
\hline \multirow{2}{*}{ Pelvi-abdominal CT } & \multicolumn{2}{|c|}{ Staging laparoscopy } & \multirow{2}{*}{ Total } & \multirow{2}{*}{ p-value } \\
\cline { 2 - 3 } & Metastasis & No metastasis & & \\
\hline Metastatic diseases & & & 8 & \multirow{2}{*}{$0.026^{*}$} \\
Metastasis & 6 & 2 & 18 & \\
No Metastasis & 4 & 14 & 8 & \multirow{2}{*}{$0.021^{*}$} \\
\hline Liver metastasis & 6 & 2 & 18 & \\
Metastasis & 2 & 16 & & \\
No metastasis & 0 & 0 & 0 & \multirow{2}{*}{$0.015^{*}$} \\
\hline Peritoneal metastasis & 2 & 24 & 26 & \\
Metastasis & & 0 & & \\
No Metastasis &
\end{tabular}

*Statistically significant at $p$ value $<0.05 ; C T=$ Computed Tomography 
When we used pelvi-abdominal $\mathrm{CT}$ to assess the metastatic spread, 18 patients were non-metastatic, whereas the other 8 patients had metastatic lesions involving the liver or peritoneum. However, when we performed staging laparoscopy on these patients, it was able to detect the presence of metastasis in 4 patients of the non-metastatic group and confirm its presence in 6 patients in the metastatic group (Table 2). Moreover, both pelvi-abdominal CT and staging laparoscopy had equally high specificity for metastatic diseases (99\%). Regarding the sensitivity, staging laparoscopy was superior to pelvi-abdominal CT in detecting the presence of metastatic disease in general ( $83 \%$ vs 66\%, $p=0.026$ ) (Table 3 ).

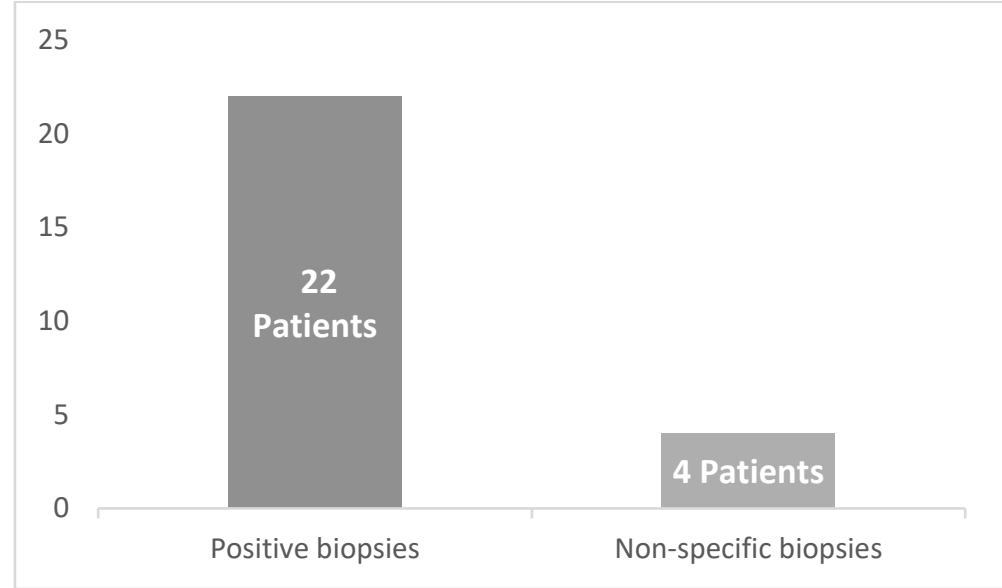

Figure 1. Colonoscopy biopsies' results

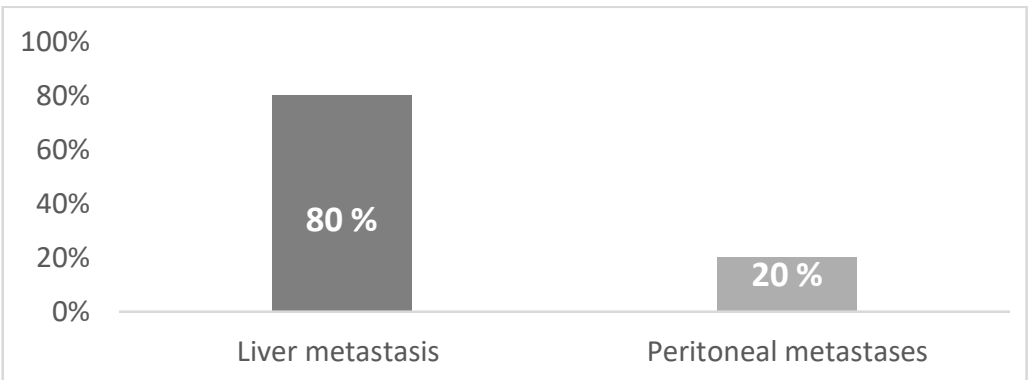

Figure 2. Pattern of metastasis in colorectal cancer

\section{Discussion}

Preoperative radiographic imaging has limitations in detecting regional invasion of the primary tumor or the presence of metastatic disease. However, staging laparoscopy plays a major role in identifying and evaluating metastasis in colorectal cancer patients ${ }^{(5)}$. In the present study, we evaluated this role thoroughly. We enrolled twenty-six patients diagnosed with colorectal cancer, all of whom had preoperative pelvi-abdominal $\mathrm{CT}$ with contrast and diagnostic laparoscopy aiming at evaluating and staging their disease. According to the results of staging laparoscopy, liver was the most frequent site for metastatic disease in 
colorectal cancer patients. This is not a novel finding and has been already established in the literature and attributed to the portal circulation. Up to $18 \%$ of colorectal cancer patients who presents for first medical consultation have liver metastasis and $10-25 \%$ are diagnosed at the time of primary tumor resection ${ }^{(6)}$. In the present study, pelvi-abdominal $\mathrm{CT}$ revealed that $69 \%$ of patients (18/26) were non-metastatic, and $31 \%$ of cases (8/26) were metastatic to the liver. Staging laparoscopy of these patients revealed metastatic spread in a total number of 10 cases (38\%), eight of these had liver me- tastases, while two patients had metastatic spread in the form of peritoneal nodules. This is consistent with the findings by Indrakumar et al, who assessed the role of staging laparoscopy in gastric tumors. They reported that diagnostic laparoscopy was effectively able to detect distant metastasis in 12 patients which were previously undetected on $\mathrm{CT}^{(7)}$. Another study on gastric tumors reported similar findings $(8)$. However, and as far to our knowledge, none of the previous studies compared between CT and laparoscopy findings in colorectal cancer patients.

Table 3: Sensitivity and specificity of staging laparoscopy and pelviabdominal $\mathrm{CT}$ in detecting metastasis. $(\mathrm{N}=26)$

\begin{tabular}{|c|c|c|c|c|c|}
\hline \multirow{2}{*}{ Metastasis } & \multicolumn{2}{|c|}{ Staging laparoscopy } & \multicolumn{2}{c|}{ Pelvi-abdominal CT } & \multirow{2}{*}{ p value } \\
\cline { 2 - 5 } & Sensitivity & Specificity & Sensitivity & Specificity & \\
\hline Metastatic diseases & $83 \%$ & $99 \%$ & $66 \%$ & $99 \%$ & $0.026^{*}$ \\
\hline
\end{tabular}

*Statistically significant at $p$ value $<0.05, \mathrm{CT}=$ Computed Tomography

We found that staging laparoscopy had $83 \%$ sensitivity and $99 \%$ specificity in detecting metastatic spread. Muntean et al found that its sensitivity in colorectal cancer metastasis was $94 \%$, with a specificity of $100 \%(9)$. The difference between the reported results between this study and ours can be due to the relatively small number of cases included in our study. As for CT scan, a study reported that $\mathrm{CT}$ had a sensitivity of $85 \%$ and a specificity of $97 \%$ in detecting liver metastases $^{(10)}$. However, in the settings of peritoneal spread, a study reported that CT was moderately sensitive in detecting peritoneal carcinomatosis but poorly sensitive for individual peritoneal tumor deposits. The study concluded that preoperative $\mathrm{CT}$ was a reliable tool in detecting the presence, size, and location of peritoneal tumor implants in patients with colorectal cancer ${ }^{(11)}$. When compar- ing staging laparoscopy to pelvi-abdominal $\mathrm{CT}$, both modalities had equally high specificities for metastasis. However, regarding their sensitivities, staging laparoscopy was superior to pelvi-abdominal $\mathrm{CT}$ in detecting the presence of metastatic disease ( $83 \%$ versus $66 \%$ ). Although this has not been tested in colorectal tumors before; yet, in gastric tumors, laparoscopy proved to be very sensitive in detecting peritoneal metastasis compared to $\mathrm{CT}$ especially in small lesions $(5 \mathrm{~mm})^{(12)}$.

Strengths and limitations:

This work, to our knowledge, is the first of its kind to compare the efficiency of staging laparoscopy and CT in detecting metastatic disease among colorectal cancer patients. However, the study was conducted at a single hospital, which resulted in a limited number of available 
patients. The small sample size might have hindered us to estimate the actual sensitivity and specificity of staging laparoscopy and $\mathrm{CT}$ as well.

\section{Conclusion}

Staging laparoscopy is superior to $\mathrm{CT}$ scan with contrast in identifying the presence of a metastatic spread in colorectal cancer patients. Future studies should be conducted on a higher number of patients in order to detect the actual value of staging laparoscopy in these settings.

Source(s) of funding: None

Conflicts of Interest: None

\section{References}

1. Ferlay J, Soerjomataram I, Ervik M, et al. GLOBOCAN 2012 v1.o, Cancer Incidence and Mortality Worldwide: IARC CancerBase No. 11. Lyon, France: International Agency for Research on Cancer. 2013;

2. Labianca R, Mosconi S, Nordlinger B, Beretta GD, Cervantes A, Arnold D. Early colon cancer: ESMO Clinical Practice Guidelines for diagnosis, treatment and follow-upt. Ann Oncol [Internet]. 2013 Oct 1;24(suppl_6):vi64vi72.

3. Macrae FA, Bendell J. Clinical presentation, diagnosis, and staging of colorectal cancer. UpToDate. 2018.

4. Society of American Gastrointestinal and Endoscopic Surgeons. Guidelines for Diagnostic Laparoscopy. 2010.

5. Marks J. Diagnostic staging laparoscopy: General principles for staging primary digestive malignancies. Up To Date. 2017.

6. Valderrama-Trevino Al, Barrera-Mera B, Ceballos-Villalva JC, Montalvo-Jave EE. Hepatic Metastasis from Colorectal Cancer. Euroasian J Hepato-Gastro- enterology. 2017; 7 (2):166-75.

7. Indrakumar A, Mandakulutur GS, Banavara KR. Role of staging laparoscopy in upstaging $\mathrm{CT}$ findings and influencing treatment decisions in gastric cancers. IJRMS 2016;4(12):52126.

8. McCulloch $P$, Johnson $M$, Jairam R, Fischer W. Laparoscopic staging of gastric cancer is safe and affects treatment strategy. Ann R Coll Surg Engl. 1998;80(6):400-2.

9. Muntean V, Oniu T, Lungoci C, Fabian O, Munteanu D, Molnar G, et al. Staging laparoscopy in digestive cancers. J Gastrointestin Liver Dis. 2009 Dec;18(4):461-7.

10. Kijima S, Sasaki T, Nagata K, Utano K, Lefor AT, Sugimot H. Preoperative evaluation of colorectal cancer using CT colonography, MRI, and PET/CT. World J Gastroenterology. 2014; 20 (45): 16964-75.

11. de Bree E, Koops W, Kröger R, et al. Peritoneal Carcinomatosis from Colorectal or Appendiceal Origin: Correlation of Preoperative CT with Intraoperative Findings and Evaluation of Interobserver Agreement. J Surg Oncol. 2004. (86) 64-73 p.

12. Singh HK, Elamurugan TP, Sreenath GS, $R$ VPN. Efficacy of contrast enhanced computed tomography and diagnostic laparoscopy in detecting unsuspected peritoneal metastasis in gastric carcinoma. Int Surg J. 2017;4(1):181-4. 